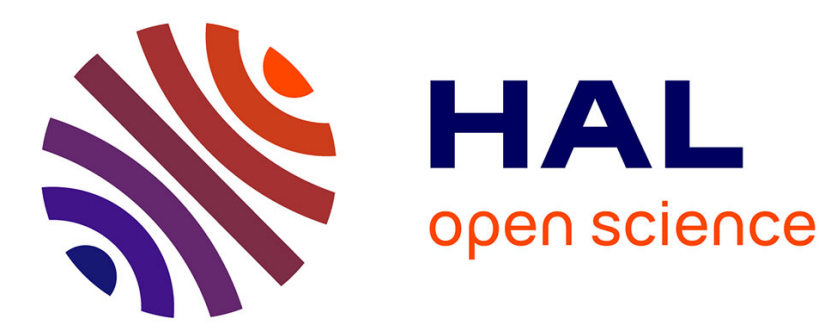

\title{
A theory of distributed aspects
}

Nicolas Tabareau

\section{- To cite this version:}

Nicolas Tabareau. A theory of distributed aspects. 2009. inria-00423996v3

\section{HAL Id: inria-00423996 https://hal.inria.fr/inria-00423996v3}

Preprint submitted on 19 Jan 2010 (v3), last revised 16 Mar 2010 (v4)

HAL is a multi-disciplinary open access archive for the deposit and dissemination of scientific research documents, whether they are published or not. The documents may come from teaching and research institutions in France or abroad, or from public or private research centers.
L'archive ouverte pluridisciplinaire HAL, est destinée au dépôt et à la diffusion de documents scientifiques de niveau recherche, publiés ou non, émanant des établissements d'enseignement et de recherche français ou étrangers, des laboratoires publics ou privés. 


\title{
A theory of distributed aspects
}

\author{
Nicolas Tabareau \\ INRIA, Ascola team \\ École des mines de Nantes, France
}

\begin{abstract}
Over the last five years, several systems have been proposed to take distribution into account in Aspect-Oriented Programming. While they appeared to be fruitful to develop or improve distributed component infrastructures or application servers, those systems are not underpinned with a formal semantics and so do not permit to establish properties on the code to be executed. This paper introduces the aspect join calculus - an aspect-oriented and distributed language based on the join calculus, a member of the $\pi$ calculus family of process calculi suitable as a programming language. It provides a first formal theory of distributed $\mathrm{AOP}$ as well as a base language in which many features of previous distributed AOP systems can be formalized.
\end{abstract}

The semantics of the aspect join calculus is given by a (chemical) operational semantics and a type system is developed to ensure properties satisfied by aspects during the execution of a process. We also give a translation of the aspect join calculus into the core join calculus. The translation is proved to be correct by a bisimilarity argument. In this way, we provide a well-defined version of a weaving algorithm which constitutes the main step towards an implementation of the aspect join calculus directly in JoCaml.

We conclude this paper by showing that despite its minimal definition, the aspect join calculus is a convenient language in which existing distributed AOP languages can be formalized. Indeed, many features (such as remote pointcut, distributed advice, migration of aspects, asynchronous and synchronous aspects, re-routing of messages and distributed control flow) can be defined in this simple language.

\section{Categories and Subject Descriptors}

D.3.1 [Programming languages]: Formal definitions and theory; D.1.3 [Programming Techniques]: Concurrent Programming - Distributed programming

\section{General Terms}

Languages, Theory

\section{INTRODUCTION}

Distributed applications are more complex to develop than sequential applications, mainly because of synchronization issues and distribution of the code across the network. It has been advocated that traditional programming languages do not allow to separate distribution concerns from standard functional concerns in a satisfactory way. For instance, data replication, transactions, security, and fault tolerance often crosscut the business code of a distributed application. Aspect-Oriented Programming (AOP) promotes better separation of concerns in software systems by introducing aspects for the modular implementation of crosscutting concerns. Indeed, AOP provides the facility to intercept the flow of control in an application and perform new computation on that execution point. In this approach, computation at certain execution points, called join points, may be intercepted by a particular condition, called pointcut, and modified by a piece of code, called advice.

But even though AOP is routinely used in distributed component infrastructures (such as EJB) or application servers (such as JBoss), most AOP systems do not support the remote definition or application of aspects. For instance, in Spring or JBoss, non-distributed aspects are used to manipulate distributed infrastructures. As pointed out by Tanter et al. [17], AOP in distributed systems is not distributed AOP. Over the past five years, distributed AOP was the focus of several AOP systems: JAC [13], DJcutter [12], ReflexD [17], AWED [11] and a Scheme-like language with distribution and aspects [16]. Those languages introduce new concepts for distributed AOP such as remote pointcut (advice triggered by remote join points), distributed advice (advice executed on a remote host), migration of aspects, asynchronous and synchronous aspects, distributed control flow. Most of those systems are based on Java and RMI in order to promote the role of AOP on commonly-used large-scale distributed applications. But the temptation of using a rich language to develop interesting applications has a fundamental drawback: it makes almost impossible the definition of a formal semantics for distributed aspects. Therefore, to date, no theory of distributed aspects has been developed.

In this paper, we propose a theory for distributed AOP based on a well formalized distributed language, the distributed join calculus [4]. The join calculus, founded on the chemical abstract machine and fully implemented in JoCaml, has been developed to fill in the gap between easy-to-reasonabout distributed languages such as the $\pi$-calculus and easy- 
to-program-with distributed languages such as Java-RMI. To stay close to the object oriented tradition of AOP, we use an object oriented and distributed variant of the join calculus. This full variant is just a mix between the objective join calculus [6] and the distributed join calculus [5]. We define a notion of aspects on this calculus and give its formal semantics by altering the main reduction rule of the join calculus. The resulting aspect join calculus is provided with a type system that guarantees safety properties such as the absence of mismatch in the number or type of arguments when an aspect returns to the original program using the keyword proceed, or the absence of host duplication in the network.

We additionally provide a translation of this aspect join calculus into the core join calculus. This translation implements the weaving algorithm that remains implicit in the abstract semantics and makes possible an implementation of the aspect join calculus directly in JoCaml. The correctness of the translation is given by a bisimilarity proof. Note that a similar approach has already been investigated in a non-distributed setting [8], where an aspect oriented language $\grave{a}$ la AspectJ is given with its operational semantics and a weaving algorithm is presented with a proof of correctness. We conclude the paper by showing how our calculus can be a cornerstone for the formulation of semantics for existing distributed oriented languages. Indeed, many features of distributed AOP languages can be defined in this simple language.

Section 2 presents the distributed and objective join calculus. Section 3 introduces the aspect join calculus. Section 4 defines the translation of the aspect join calculus into the core join calculus. Section 5 shows how basic concepts of distributed AOP can be described in the aspect join calculus. The reader that wants a quick overview of the aspect join calculus should only read Sections 3 and 5 .

\section{THE DISTRIBUTED OBJECTIVE JOIN-CALCULUS}

The original version of the join calculus is a simple namepassing calculus related to the $\pi$-calculus but with a functional flavor $[4,5]$. In this calculus, communication channels are statically defined: channels are created together with a set of reaction rules that specify, once and for all, how messages sent on these names will be synchronized and processed. We decide here to present an object-oriented version of the join-calculus [6] with an explicit notion of location to account for distribution [5].

\subsection{Message passing and internal states}

Before going into the details of the distributed objective join calculus, we begin with the example of the class buffer presented in [6]. The basic operation of the join-calculus is asynchronous message passing and, accordingly, the definition of an object describes how messages received on some labels can trigger processes. For instance, the term

obj continuation $=$ reply ()$\triangleright$ out.print_int $(n)$

defines an object that reacts to messages on its own label reply by sending a message with label print_int and con-

\begin{tabular}{|c|c|}
\hline $\begin{array}{l}\mathrm{fn}(l(\vec{v})) \\
\mathrm{fn}\left(M \& M^{\prime}\right) \\
\mathrm{fn}(M \triangleright P) \\
\mathrm{fn}\left(D \text { or } D^{\prime}\right) \\
\mathrm{fn}(H[D: P]) \\
\mathrm{fn}(c) \\
\mathrm{fn}(0) \\
\mathrm{fn}(\operatorname{self}(z) D) \\
\mathrm{fn}(x \cdot M) \\
\mathrm{fn}(\mathrm{goH} H) \\
\mathrm{fn}(P \& Q) \\
\mathrm{fn}(\text { obj } x=C \text { init } P \text { in } Q) \\
\mathrm{fn}(\text { class } c=C \text { in } P)\end{array}$ & $\begin{array}{l}=\left\{v_{i} / i \in I\right\} \\
=\mathrm{fn}(M) \cup \mathrm{fn}\left(M^{\prime}\right) \\
=\mathrm{fn}(P) \backslash \mathrm{fn}(M) \\
=\mathrm{fn}(D) \cup \mathrm{fn}\left(D^{\prime}\right) \\
=\mathrm{fn}(D) \cup \mathrm{fn}(P) \\
=\{c\} \\
=\emptyset \\
=\mathrm{fn}(D) \backslash\{z\} \\
=\{x\} \cup \mathrm{fn}(M) \\
=\mathrm{fn}(P) \\
=\mathrm{fn}(P) \cup \mathrm{fn}(Q) \\
=(\mathrm{fn}(C) \cup \mathrm{fn}(P) \cup \mathrm{fn}(Q)) \backslash\{x\} \\
=\mathrm{fn}(C) \cup(\mathrm{fn}(P) \backslash\{c\})\end{array}$ \\
\hline
\end{tabular}

Figure 1: Definition of free names $\mathrm{fn}(\cdot)$

tent $n$ to an object named out that prints integers on the terminal.

But labels may also convey messages representing the internal state of an object, rather than an external method call. This is the case of label some in the following definition of a buffer :

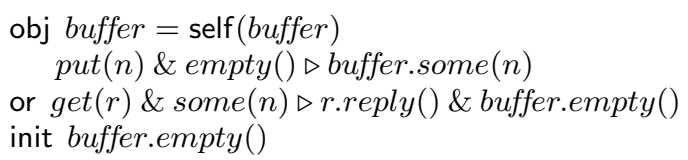

Such a buffer can either be empty or contain one element. The state is encoded as a message pending on empty or some, respectively. Object buffer is created empty, by sending a first message on empty in the (optional) init part of the obj construct. In the definition of an object, the ' $\triangleright$ ' symbol defines a reaction rule that consumes the messages on its left hand side and produces the messages on its right hand side.

In our definition of pointcut for distributed aspects, we will have to consider labels that are common to different objects. This will be the case if we want to define a replication buffer aspect that will intercept the synchronization on the label get on any buffer object. This means that we need a notion of class instantiations. A buffer can then be defined as the instantiation of a class buffer:

$$
\begin{aligned}
& \text { class buffer }=\operatorname{self}(z) \\
& \quad \operatorname{put}(n) \& \operatorname{empty}() \triangleright z . \operatorname{some}(n) \\
& \text { or get }(r) \& \operatorname{some}(n) \triangleright \operatorname{rreply}() \text { \& z.empty }() \\
& \text { in obj } b=\text { buffer init b.empty }()
\end{aligned}
$$

Note that to keep the buffer object consistent, there should be a single message pending on either empty or some. This remains true as long as external processes cannot send messages on these labels directly. This can be enforced by a privacy discipline, as described in [6], that is not addressed in this paper.

\subsection{Syntax}

We use four disjoint countable sets of identifiers for object names $x, y, z \in \mathcal{O}$, classes $c \in \mathcal{C}$, labels $l \in \mathcal{L}$ and hosts $H \in \mathcal{H}$. Tuples are written $\left(v_{i}\right)^{i \in I}$ or simply $\vec{v}$. The gram- 


\begin{tabular}{|c|c|c|}
\hline$P::=$ & $\begin{array}{l}0 \\
x . M \\
\text { go } H ; P \\
P \& P \\
\text { obj } x=C \text { init } P \text { in } P \\
\text { class } c=C \text { in } P\end{array}$ & $\begin{array}{l}\text { Processes } \\
\text { null process } \\
\text { message sending } \\
\text { migration request (to host } H \text { ) } \\
\text { parallel composition } \\
\text { object definition } \\
\text { class definition }\end{array}$ \\
\hline$C::=$ & $\begin{array}{l}c \\
\operatorname{self}(z) D\end{array}$ & $\begin{array}{l}\text { Classes } \\
\text { class variable } \\
\text { self binding }\end{array}$ \\
\hline$D::=$ & $\begin{array}{l}M \triangleright P \\
H[D: P] \\
D \text { or } D\end{array}$ & $\begin{array}{l}\text { Definitions } \\
\text { reaction rule } \\
\text { sub-location (named } H) \\
\text { disjunction }\end{array}$ \\
\hline$M::=$ & $\begin{array}{l}l(\vec{v}) \\
M \& M\end{array}$ & $\begin{array}{l}\text { Patterns } \\
\text { message } \\
\text { synchronization }\end{array}$ \\
\hline
\end{tabular}

Figure 2: Syntax for the core objective join calculus

mar of the distributive objective join calculus is given in Figure 2 ; it has syntactic categories for processes $P$, classes $C$, definitions $D$, and patterns $M$. A reaction rule $M \triangleright P$ associates a pattern $M$ with a guarded process $P$. Every message pattern $l(\vec{v})$ in $M$ binds the object names $\vec{v}$ with scope $P$. In the join-calculus, it is required that every pattern $M$ guarding a reaction rule be linear, that is, labels and names appear at most once in $M$. Class definitions class $c=C$ in $P$ are the only binders for class names $c$, with scope $\mathrm{P}$. The scoping rules appear in Figure 1. In addition, the object definition obj $x=C$ init $P$ in $Q$ binds the name $x$ to $C$. The scope of $x$ is every guarded process in $C$ (here $x$ means "self") and the processes $P$ and $Q$. The term $H[D: P]$ hosts the definition $D$ and process $P$ at location $H$. Migration request is described by go $H ; P$. Free names in processes and definitions, written $\mathrm{fn}(\cdot)$, are defined accordingly; a formal definition of free names appears in Figure 1. We suppose that class name definitions are unique. Objects are taken modulo renaming of bound names (or $\alpha$-conversion).

\subsection{Semantics}

The operational semantics is given as a reflexive chemical abstract machine [4]. A machine $\mathcal{D} \Vdash^{\varphi} \mathcal{P}$ consists of a set of named definitions $\mathcal{D}$ (representing objects in the machine) and of a multiset of processes $\mathcal{P}$ running in parallel at location $\varphi=H_{1} \cdots H_{n}$. Each rewrite rule applies to configurations, that is a set of machines running in parallel

$$
\mathcal{D}_{1} \Vdash^{\varphi_{1}} \mathcal{P}_{1} \quad\|\quad \cdots \quad\| \quad \mathcal{D}_{n} \Vdash^{\varphi_{n}} \mathcal{P}_{n}
$$

(usually called chemical solutions). Intuitively, a root location $H$ can thought of as an IP address on a network and a machine at location $H$ can be thought of as a physical machine at this address. Differently, a machine at sublocation $H H^{\prime}$ can be thought of as a process executed on a physical machine (whose location is $H$ ). This includes for example the treatment of several threads, or of multiple virtual machines executing on the same physical machine. We write $x . D$ for a named definition in $\mathcal{D}$, and always assume that there is at most one definition for $x$ in $\mathcal{D}$. Chemical reduc- tions are obtained by composing rewrite rules of two kinds: structural rules $\equiv$ represent the syntactical rearrangement of terms; reduction rules $\longrightarrow$ represent the basic computation steps. The rules for the objective join calculus are given in Figure 3, with side conditions for rule RED: $\sigma$ is a substitution with domain $\mathrm{fn}(M)$; the processes $M \sigma$ and $P \sigma$ denote the results of applying $\sigma$ to $M$ and $P$, respectively.

Rules PAR and NIL make parallel composition of processes associative and commutative, with unit 0 . Rule OBJ describes the introduction of an object (up-to $\alpha$-conversion, we can consider that any definition of an object $x$ appears only once in a configuration). Rule JoIn gathers messages sent to the same object. Rule RED states how messages can be jointly consumed and replaced by a copy of a guarded process, in which the contents of these messages are substituted for the formal parameters of the pattern. In chemical semantics, each rule usually mentions only the components that participate to the rewriting, while the rewriting applies to every chemical solution that contains them. More explicitly, we provide two context rules CONTEXT and CONTEXTObJ. In Rule Context, the symbol $\longrightarrow / \equiv$ stands for either $\longrightarrow$ or $\equiv$ (the same in premise and conclusion). In Rule Context-OBJ, the side condition $x \notin \mathrm{fn}(D) \cup \mathrm{fn}(P)$ prevents name capture when introducing new objects (the sets $\mathrm{fn}(D)$ and $\mathrm{fn}(P)$ are defined in Figure 1). Rule Comm is reminiscent of distributed systems, where routing is a different step from actual computation. This rule states that a message emitted in a given location $\varphi$ on a channel name $x$ that is remotely defined can be forwarded to the machine at location $\psi$ that contains the definition of $x$. Later on, this message can be used within $\psi$ to assemble a pattern of messages and to consume it locally, using a local RED step. Rule LOC introduces a new machine at sub-location $H$ of $\varphi$ with $D$ as initial definitions and $P$ as initial processes. The side condition "H frozen" means that there is no other machine of the form $\Vdash^{\varphi H \psi}$ in the configuration. The notation $\{x . D\}$ and $\{P\}$ states that there are no extra definitions or processes at location $\varphi H$. Finally, rule Move gives the 
semantics of migration. A sub-location $\varphi H_{1}$ of $\varphi$ wants to move to a sub-location $\psi H_{2}$ of $\psi$. On the right hand side, the machine $\mathbb{H}^{\varphi}$ is fully discharged of the location $H_{1}$. Note that $P$ can be executed at any time, whereas $Q$ can only be executed after the migration.

In the following, we consider only configurations where every name is defined in at most one machine (or local solution). This condition is preserved by the semantics, and simplifies the usage of rule Cомм: for every message, the rule applies at most once, and delivers the message to a unique receiving location.

\subsection{An encoding of (a part of) Java}

The objective join calculus is inherently asynchronous. Nevertheless, it is folklore that synchronous calls can be encoded by use of continuations.

For instance, we can encode a large part of Java (without inheritance) in the objective join calculus. Fields are translated into labels containing the value of the field (as for labels some and empty of the class buffer). An expression

$$
\mathrm{x} \cdot \mathrm{m}(\overrightarrow{\mathrm{v}}) ; \mathrm{P}
$$

is translated into

$$
\text { obj } k=\operatorname{return}() \triangleright P \text { in } m(k, \vec{v})
$$

A method

$$
\mathrm{m}(\overrightarrow{\mathrm{v}})\{\text { return e\} }
$$

using fields $f_{1}, \ldots, f_{n}$ is translated into the reaction rule

$$
m(k, \vec{v}) \&\left(f_{i}\left(x_{i}\right)\right)_{i \in I} \triangleright k \text {.return }(e) \&\left(f_{i}\left(x_{i}\right)\right)_{i \in I}
$$

where $k$ is the explicit continuation passed to $m$ and $e$ is a base expression. To illustrate this, we present the translation of the resuming example of the seminal paper on Featherweight Java [7]. This example can be written, in absence of inheritance, as:

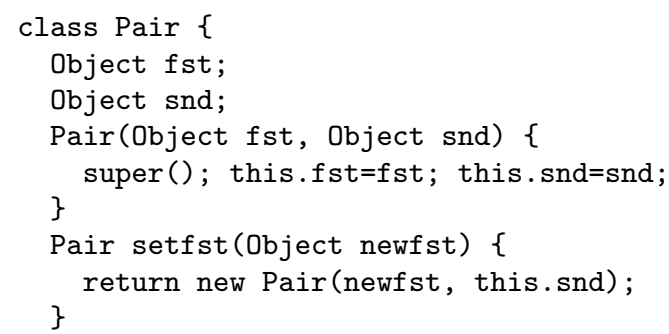

The class Pair has two fields fst and snd, an initialization method (also written Pair) and a method setfst that returns a new Pair with different first element. In the join calculus, this class becomes:

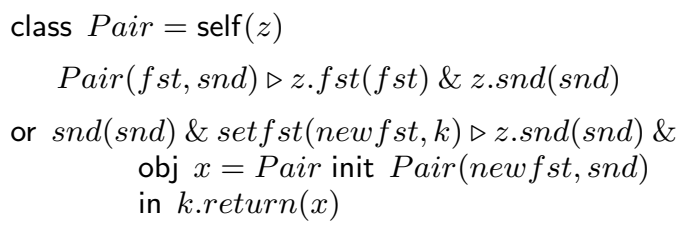

The two fields have been translated in two labels $f s t$ and snd. These two labels are used in a synchronization pattern to communicate the internal value of the fields. On the right hand side of a rule, those labels have to be present again to maintain the value of the fields in the local solution. The initialization is performed by the reaction rules Pair $(f s t, s n d) \triangleright$ $z . f s t(f s t) \& z . s n d(s n d)$. The method setfst is now seen as a reaction that synchronizes the label set $f$ st with the label snd (to get the value of the field snd) and then produces the label snd again and creates a new pair object that is passed to the continuation $k$. So the continuation comes in the picture when a method returns.

Naturally, we can encode much more than Featherweight Java, the pure functional part of Java. As sketched in Section 2.1 with the class buffer, it is also possible to capture the imperative flavour of Java by encoding internal states with labels. For instance, we refer the interested reader to [5] for a description of references in the join calculus.

Note that if we add inheritance in the join calculus, as done in [6], then most of Java can be encoded.

\subsection{Migration in the calculus}

In contrast with some models of distributed systems [14], the explicit routing of messages is not described by the calculus. Rule Comm applies at most once and delivers the message to a unique receiving location. Since every object is located, the interpretation of the remote object mechanism of JavaRMI, implemented using stub and skeleton, is transparent.

Rule Move says that the migration process on the network is based on sub-locations but not objects nor processes. When a process decides to move, it moves with all the definitions and processes present at the same sub-location. Nevertheless, we can encode object/process migration by defining a fresh sub-location and uniquely attaching an object/process to it. Then the migration of the sub-location will be equivalent to the migration of the object/process.

In contrast to Java-RMI, there is no mechanism of serialization, with copy before migration. Jeffrey has considered a distributed object calculus where serialization is explicit [9]. Nevertheless, the continuation passing style encoding presented above is close to the semantics given by Ahern and Yoshida for method invocation in a core Java with RMI [1]. Indeed, most of the mechanism of Java-RMI (as formalized in [1]) can be encoded in the distributed join calculus. One just has to create a new location for each object such that the migration of an object becomes the migration of the sublocation attached to this object. So except from serialization concerns, the distributed join calculus and Java-RMI have the same flavour.

\subsection{Typing}

The grammar of type expressions is given by

$$
\begin{aligned}
& A::=\text { int } \mid \text { bool } \mid[B] \\
& B::=\emptyset \mid l: \vec{A} ; B
\end{aligned}
$$

We build types out of the two base types int for natural numbers and bool for booleans. As we want to keep our core language and typing derivation as simple as possible, 


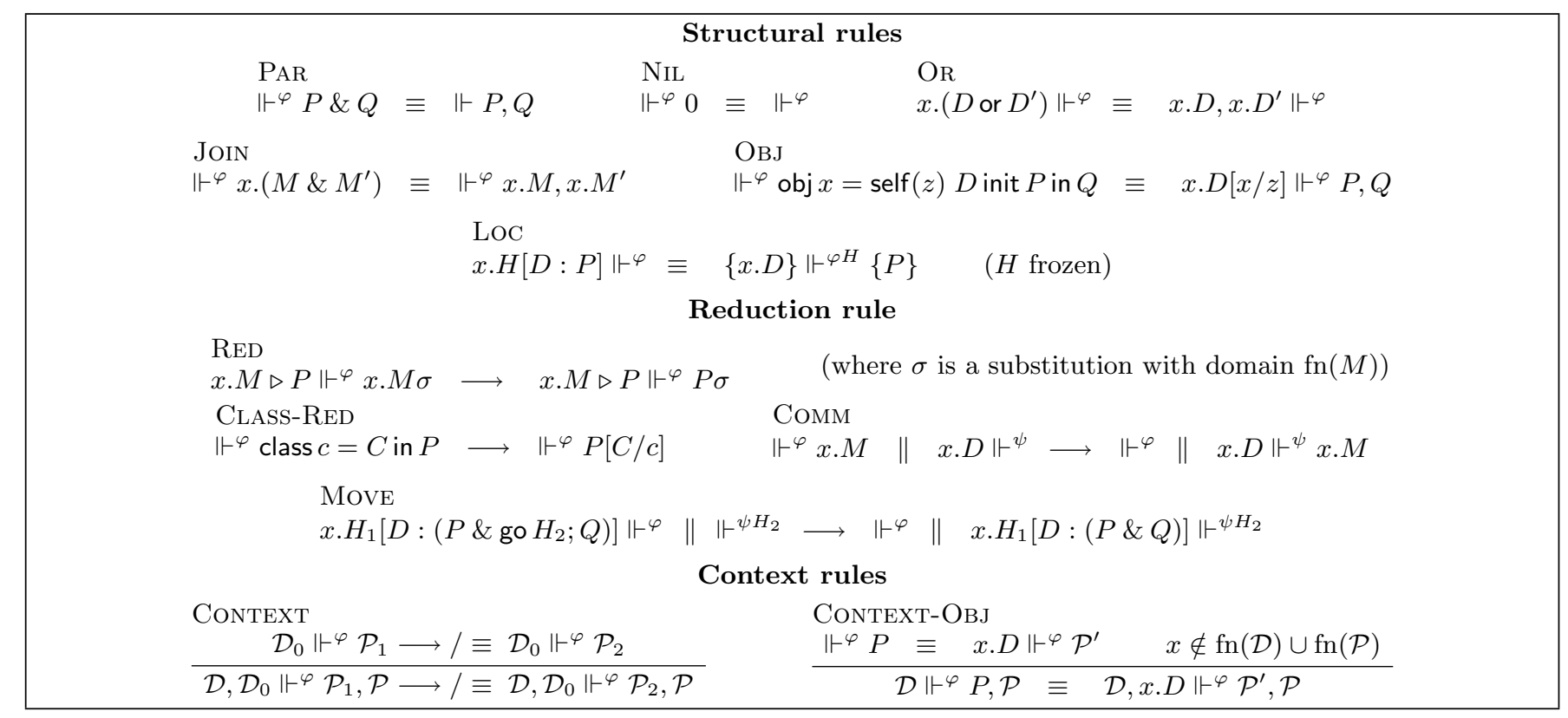

Figure 3: Chemical semantics

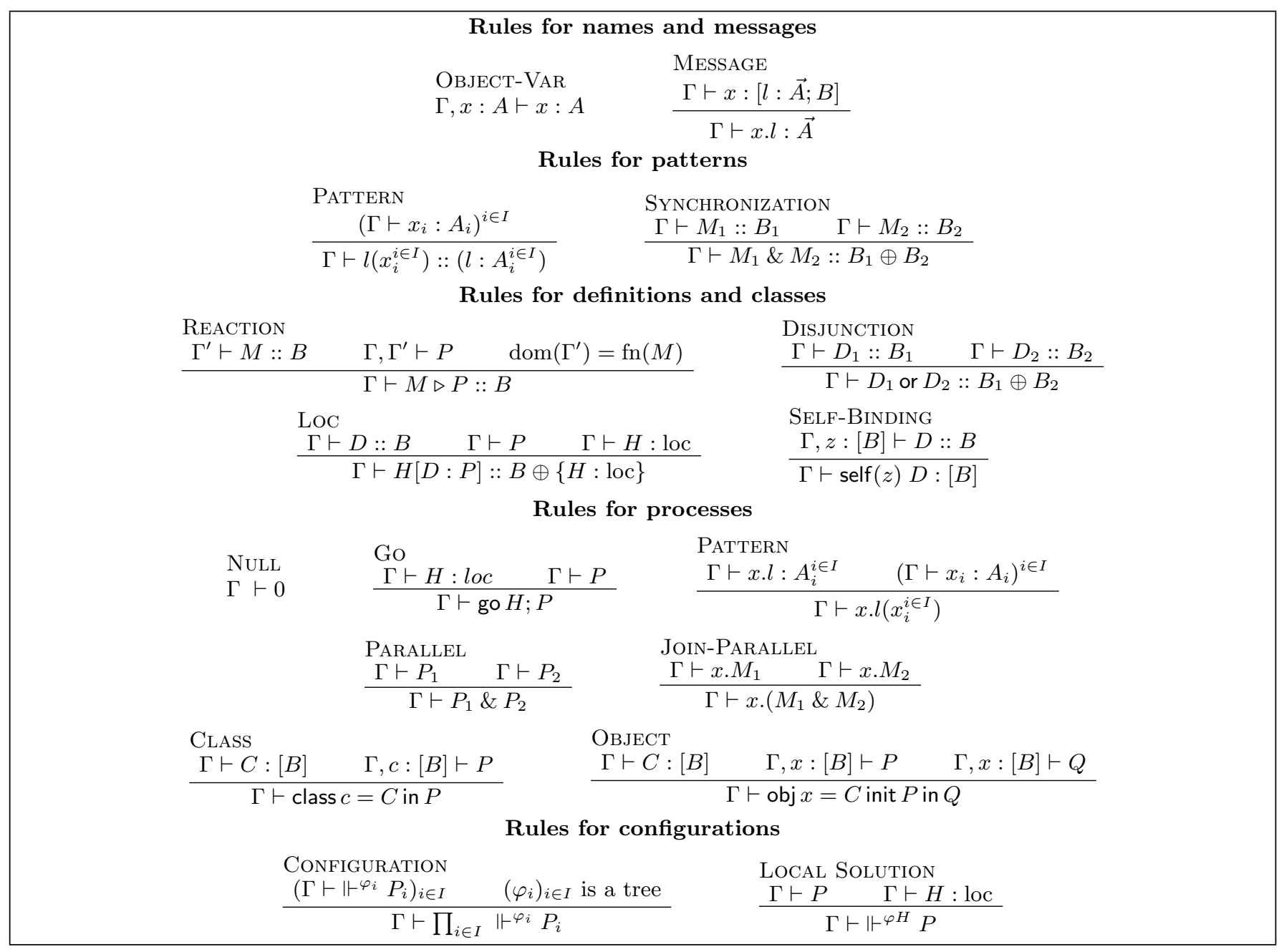

Figure 4: Typing rules 
we do not consider polymorphism in this paper, although polymorphism à la $M L$ can be defined without difficulty [6].

Object types $[B]$ collect the types of labels. For instance, the type of the object continuation is

$$
\text { continuation : [reply : int }]
$$

and the object buffer can be typed for example with

buffer : [put : int; empty : (); get: [reply : int]; some : int].

Again, the absence of polymorphism certainly make this type system look a bit odd. Indeed, one could expect the type system to be able to type the object buffer with the polymorphic type

$$
\forall \alpha, \beta:[\text { put : } \alpha ; \text { empty : ();get : [reply : } \alpha ; \beta] ; \text { some }: \alpha] \text {. }
$$

We accept this peculiar nature for the sake of simplicity. The typing judgements differ on the nature of the term in the following manner:

$$
\begin{array}{ll}
\Gamma \vdash x: A & \text { the object } x \text { has type } A \text { in environment } \Gamma \\
\Gamma \vdash x \cdot l: \vec{A} & \text { the label } l \text { conveys messages of type } \vec{A} \text { in } \Gamma \\
\Gamma \vdash M:: B & \text { the pattern } M \text { binds variables well-typed } \\
& \text { in } \Gamma \text { and joins labels in } B \\
\Gamma \vdash c:[B] & \text { the class } c \text { declares the labels of } B \text { in } \Gamma \\
\Gamma \vdash P & \text { the process } P \text { is well-typed in } \Gamma
\end{array}
$$

As usual in typing of object oriented languages (see for instance [7]), we make use of a class table $C T$ that collects all class definitions. To lighten the notation in what follows, we always assume a fixed class table $C T$. We do not describe in detail the typing rules given in Figure 4 (where we write $B_{1} \oplus B_{2}$ for the union of $B_{1}$ and $B_{2}$, with the statement that $B_{1}$ and $B_{2}$ coincide on their common labels) and we refer the reader to $[5,6]$ for more details.

Note that rules Configuration and Local Solution are only defined in case of configurations of the form $\Vdash^{\varphi} P$. Upto structural congruence, a more general rule can be easily deduced.

\subsection{Safety property}

We now present the interest of types with respect to the chemical semantics. Namely, the type system ensures that no well-typed configurations can present a runtime failure in the sense defined below. To state this safety property, we first need subject reduction for our type system.

TheOREM 1 (SUBJeCt REDUCtion). Chemical reductions preserve typing : let $\mathcal{C}$ be configuration

$$
\mathcal{C}=\mathcal{D}_{1} \Vdash^{\varphi_{1}} \mathcal{P}_{1}\|\cdots\| \mathcal{D}_{n} \Vdash^{\varphi_{n}} \mathcal{P}_{n},
$$

$\Gamma$ an environment. If $\Gamma \vdash \mathcal{C}$ and $\mathcal{C} \equiv \mathcal{C}^{\prime}$ or $\mathcal{C} \longrightarrow \mathcal{C}^{\prime}$, then there exists an environment $\Gamma^{\prime}$ such that $\Gamma^{\prime} \vdash \mathcal{C}^{\prime}$.

Proof. The proof goes by induction on structural and reduction rules. A detailed description of this induction (except for locations) can be found in [6], Appendix B. A formal (and more general) treatment of location can be found in $[15]$.

Runtime failure: We say that a configuration $\mathcal{C}$ fails when one of the following holds:
- Arity mismatch: for some message $l(\vec{v})$ in $\mathcal{P}_{i}, l(\vec{u})$ appears in a pattern of $\mathcal{D}_{j}$ with different arities or types for $\vec{v}$ and $\vec{u}$.

- Host duplichtion: there are two machines at the same location, for instance $\mathcal{D}_{i} \Vdash^{\varphi H} \mathcal{P}_{i}$ and $\mathcal{D}_{j} \Vdash^{\psi H} \mathcal{P}_{j}$

- Impossible migration: there is a process go $H ; P$ where $H$ is not a location name.

The first runtime failure is usual and does not rely on distribution. It is about method invocation with a right number of arguments and the right types. The second one models IP address duplication. Avoiding this duplication in a configuration makes the semantics of migration unambiguous. The last failure corresponds to a wrong IP address when trying to move a process to another hosting machine.

$$
\text { fail. }
$$

TheOREM 2 (SAFETY). Well-typed configurations do not

Proof. We check that no failure listed above can occur for a well-typed configurations. The conclusion then follows from subject reduction. This kind of proof is standard and a similar detailed proof can be found in [15].

\subsection{Basic extension of the language}

To make the definition of processes more digestible in the rest of the paper, we flavor our language with some common primitive. We add a notion of string and list of strings, equality test on strings, a traditional let binding, a conditional if then else instruction and a particular variable localhost that represents the current location on which a process is executing. Except for equality testing, all those constructions can be easily encoded into the join-calculus. A string is written "name", and a list is written $\left[l_{1} ; \ldots ; l_{n}\right]$ with concatenation noted $L_{1} \cdot L_{2}$, constructor $l:: L$ and empty list [].

Since the join-calculus has lexical scoping, programs being executed on different machines do not initially share any port name; therefore, they would normally not be able to interact with one another. To bootstrap a distributed computation, it is necessary to exchange a few names, and this is achieved using a built-in library called the name server. Once this is done, these first names can be used to communicate some more names and to build more complex communication patterns.

The interface of the name server mostly consists of two functions to register and look up arbitrary values in a "global table" indexed by plain strings. A process that wants to associate the string "foo" to the name $x$ simply executes ns.register ( $x$, "foo"). Then, an object $y$ on a remote location can ask to the name server which name is associated to "foo" by executing ns.lookup("foo", y, return). The name server will then send the name $x$ on y.return.

\section{A JOIN CALCULUS WITH ASPECTS}

In Java, the basic event of the language is the call of a method. In ML, the basic event of the language is the application of a function. Therefore, it is not surprising to see those events as basic blocks for the definition of join points 


\begin{tabular}{|c|c|c|}
\hline$P c::=$ & $\begin{array}{l}\operatorname{rule}(c \cdot M) \\
P c \wedge P c^{o p t}\end{array}$ & $\begin{array}{l}\text { Pointcuts } \\
\text { call, arguments } \\
\text { conjunction }\end{array}$ \\
\hline$P c^{o p t}::=$ & $\begin{array}{l}\text { flow }(l) \\
\operatorname{host}(H) \\
\neg P c^{o p t} \\
P c^{o p t} \wedge P c^{o p t}\end{array}$ & $\begin{array}{l}\text { Optional Pointcuts } \\
\text { control on flow } \\
\text { control on host } \\
\text { negation } \\
\text { conjunction }\end{array}$ \\
\hline$A d::=$ & $\begin{array}{l}\ldots \\
\operatorname{proceed}(\vec{v})\end{array}$ & $\begin{array}{l}\text { Advice bodies } \\
\text { other process definitions } \\
\text { proceed }\end{array}$ \\
\hline$A::=$ & $\begin{array}{l}P c\{A d\} \\
\text { aspect } a=C \text { init } P \text { intercept }\left(P c_{i}\left\{A d_{i}\right\}\right)_{i \in I}\end{array}$ & $\begin{array}{l}\text { Advice and aspects } \\
\text { advice definition } \\
\text { aspect definition }\end{array}$ \\
\hline
\end{tabular}

Figure 5: Syntax for distributed aspects

in AspectJ [10] or AspectML [18]. To define the notion of aspects in the objective join calculus, we must understand what is a basic event of this language. It makes no doubt that it consists in the application of Rule RED to a synchronization pattern $M$. So a pointcut in the aspect join calculus will rely on a synchronization pattern.

Before going into the details of the syntax, we present a basic example of distributed aspects as defined in the language AWED [11], and show how we want to define them in aspect join calculus. In AWED, one likes to define an aspect for buffer replication in the following manner :

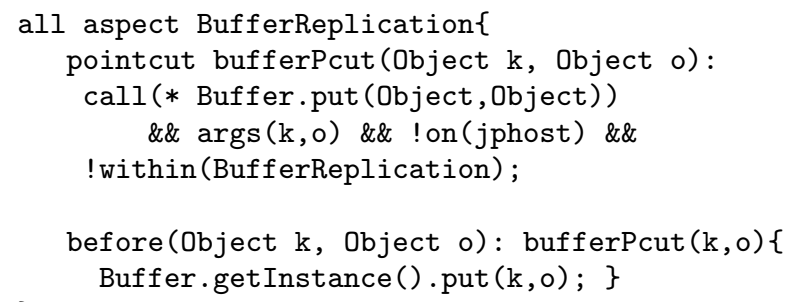

This aspect realizes a replication of the buffer each time the method buffer.put is called. The replication takes place on every machine except the machine where the method put has been caught (!on(jphost)). To prevent from an infinite replication of buffers, the condition !within (buffer Repl) guarantees that the join point is not inside an execution of the aspect buffer Repl. In our setting, using the definition of the class buffer given in Section 2.1, we can write a similar aspect:

$$
\begin{aligned}
& \mathbb{H}^{\varphi} \text { aspect } \text { buffer Repl }= \\
& \text { intercept : rule }(\text { buffer. }(\operatorname{put}(n) \& \operatorname{empty}())) \wedge \neg \operatorname{host}(\varphi) \\
& \{\text { obj } b=\text { buffer init b.empty }() \text { in }(\text { b.put }(n) \& \operatorname{proceed}(n))\}
\end{aligned}
$$

The join point now relies on the interception of the synchronization pattern put $(n) \& \operatorname{empty}()$ of the class buffer. The advice body makes an explicit use of the keyword proceed. This is because Before advice does not exist in the aspect join calculus. Indeed, in a asynchronous setting, there is no notion of before or after the execution of a method body, so the only possible advice is something that looks like the Around advice of AspectJ. Note that it might seem unsatisfactory to define an aspect by explicitly mentioning the channel empty. This can be handled with a careful management of privacy that we don't want to consider here. The basic idea is to say that an aspect should only mention public labels and will be implicitly quantified over all private labels. In that setting, the pattern of interest for the advice aspect above would simply be defined by rule(buffer.put $(n))$.

The condition $\neg$ host $(\varphi)$ guarantees that the replication does not hold when the reaction is taking place on a sub-location of the location where the aspect has been hosted. In particular, this prevents the aspect to be deploy on its own invocation of method put.

So this single aspect behaves as a single aspect BufferReplication. Nevertheless, if one tries to define such an aspect on each host of interest, then the aspects will interfere and recursively copy the buffer copied by the other aspect. In AWED, the way we can prevent this livelock to append is by the use of within. Unfortunately, this notion of "within the execution of an aspect" makes no sense in an asynchronous setting. Nevertheless, we will see later that within can be encoded with flow when we are in a fully synchronous setting.

\subsection{Syntax}

Figure 5 presents the syntax for distributed aspects in the objective join calculus. We use a countable set of identifiers for aspect names $a \in \mathcal{A}$,

An aspect aspect $a=C$ init $P$ intercept $\left(P c_{i}\left\{A d_{i}\right\}\right)_{i \in I}$ consists in a class definition $C$, an initialization process $P$ and a list of advice $P c_{i}\left\{A d_{i}\right\}$. The class $C$ and process $P$ are here to define inner fields and methods of an aspect seen as an object. Advice is defined by a pointcut $P c$ and an advice body $A d$.

A pointcut is defined by a term rule(c.M) that selects any 
reaction rule that has the pattern $M$ of the class $c$ as left hand part. A pointcut can also be defined by conditions on the history of reaction rule (flow), on the host where the join point has been selected (host). Finally, a pointcut can be constructed by negations and conjunctions of those two conditions. Note that in contrast to AspectJ, we do not need to type the intercepted pattern in rule $(c . M)$ as we explicitly mention the class to which $M$ belongs.

An advice body $A d$ is a process to be executed when the rule is intercepted. This process may contain the special keyword proceed. Definition of processes are extended with advice and aspects.

\subsection{Semantics}

Figure 6 presents the semantics of aspects. All rules of Figure 3 are conserved, expect for Rule RED that is split in two rules. Rule Asp describes the introduction of an aspect. It is similar to Rule OBJ. Rule ADV corresponds to the activation of an advice. Note that activation of advice is asynchronous.

Rule RED/AsP defines the modification of Rule RED in presence of aspects. If an advice definition $P c\{A d\}$ has a pointcut $P c$ that is satisfied, then the advice $A d$ is applied while substituting the process $P$ for the keyword proceed. Note that all pieces of advice that have a satisfied pointcut are executed in parallel. Another choice, maybe more natural with respect to the join calculus, would have been to choose one advice non-deterministically. We have chosen this definition because it offers the possibility to define a weaving algorithm that produces a configuration which is bisimilar to the original configuration (see Section 4), a very strong connection. With the non-deterministic version, we can only get a coupled bisimulation, which is weaker and would have lead to useless complications in this article.

The side condition of this rule is that $P c_{1}, \ldots, P c_{n}$ are all the pointcuts that are satisfied at this join point. For a given pointcut $P c$, this means that it intercepts the right pattern and that the condition $P c^{o p t}$ is satisfied. We just describe informally the semantics of the optional condition of a pointcut as this is not the purpose of this article. The proposition flow $(l)$ is satisfied when the message $l$ appears in the reaction tree of the intercepted reaction rule. The proposition $\operatorname{host}(H)$ is satisfied when the intercepted reaction rule is executed on a sub-location of $H$.

Rule RED/No ASP is a direct reminiscence of Rule RED in case where no aspect can be deployed.

\subsection{Typing rules}

We do not type pointcuts as they are just boolean expressions that describe the applicability of an advice.

Figure 7 introduces the two new rules required to type the aspect join calculus. Rule AsPECT is similar to Rule OBJECT, it further checks that all pieces of advice are welltyped. Rule ADVICE checks that the body of the advice is well typed once we have substituted the keyword proceed by the pattern $x . M$ of any object $x$ of the class $c$.

Of course, substituting proceed by the pattern $x . M$ is not correct with respect to the semantics of proceed. Indeed, proceed should rather be replaced by the right hand of the reaction rule involving $M$. Nevertheless, as one consider typing judgment only, this substitution is safe and makes the typing derivation easier to define.

We get subject reduction and a safety theorem similar to Theorem 2. In particular, we have the following corollary, which is not the case in AspectJ (a recent work has been done to solve this problem using type ranges [3]),

Corollary 1. A well-typed configuration makes use of proceed with the good number and types of arguments.

\section{REDUCTION TO THE CORE CALCULUS}

In this section, we present a translation of the aspect join calculus into the core join calculus. In this way, we give an implementation of the weaving algorithm with a bisimilarity proof that this translation has the same behaviour than the original configuration with aspects. A non-objective version of the aspect join calculus can thus be implemented directly in Jocaml (http://jocaml.inria.fr/), a combination of Ocaml and the join calculus. It will provide an expressive distributed AOP platform that enables formal reasoning about aspect properties. This implementation has not been developed yet and should be the subject of a future work.

Given a typed aspect join calculus configuration

$$
\emptyset \vdash\left(\Vdash^{\varphi_{1}} P_{1}\right)\|\cdots\|\left(\Vdash^{\varphi_{n}} P_{n}\right),
$$

we construct a distributed join calculus configuration without aspects by translating processes and aspects and introducing a weaver process $W$

$$
\emptyset \vdash\left(\Vdash^{\varphi_{1}} \llbracket P_{1} \rrbracket\right)\|\cdots\|\left(\Vdash^{\varphi_{n}} \llbracket P_{n} \rrbracket\right) \|\left(\Vdash^{H_{W}} W\right) .
$$

The idea is to introduce an explicit join point in every reaction rule. This join point triggers a protocol with the weaver to decide whether advice could intercept the rule and be deployed. To make the weaver able to decide if an advice can be deployed or not, the weaver must know about the history of execution. We realize this by passing the list of previous emitted messages has an argument all over the execution.

\subsection{The translation of processes}

The translation of processes is quite straightforward. Any reaction rule $M \triangleright P$ is replaced by a call to the weaver and a return method that performs the actual computation $P$. The flow of previous synchronized labels is passed as an argument for every label. For example, the class buffer would by translated to

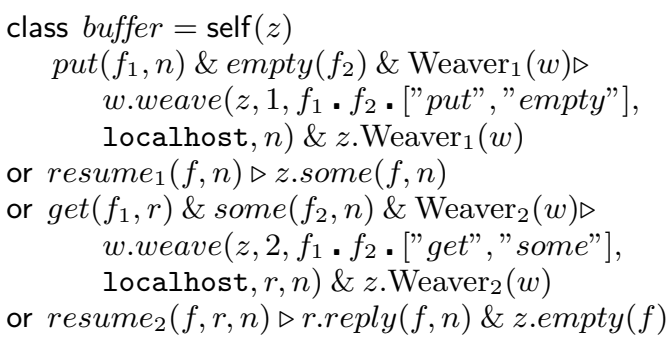




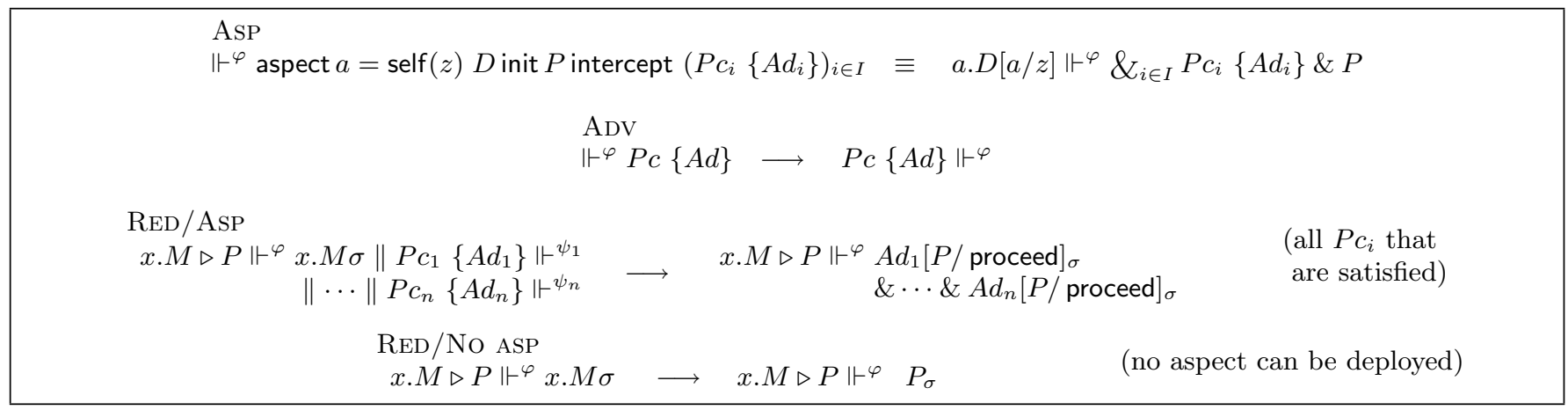

Figure 6: Semantics of aspects

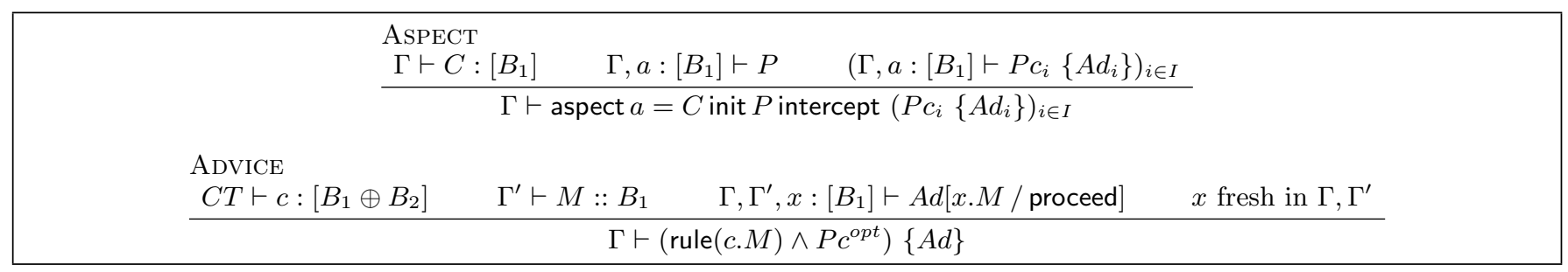

Figure 7: Typing rules for aspect join calculus

Each reaction rule of the class buffer has been divided into two reactions. The first one sends a message to the weaver with label weave. The original reaction is blocked. When an advice does a proceed (in case of Rule RED/ASP) or when the weaver detects that no aspect can be deployed (in case of Rule RED/No AsP), the message resume 1 is sent to buffer and the original reaction is performed (with potentially new arguments). The flow of performed reactions is passed along reaction rules with the used of dedicated variable $f_{1}, f_{2}, \ldots$ The location were the reaction rule is performed is sent using the localhost keyword. The location of the weaver is stored in the label Weaver.

Figure 8 describes the details of the translation for processes. Note that each object initializes its own labels Weaver $_{M}$ for each pattern $M$ appearing in $C$. To construct the flow information, we use the function listof that builds a list of messages from a pattern and an object.

$$
\begin{gathered}
\text { listof }(l(\vec{v}), x)=\left[" x . l\left(\mathrm{v}_{1}, \ldots, \mathrm{v}_{\mathrm{n}}\right) "\right] \\
\text { listof }\left(M_{1} \& M_{2}, x\right)=\operatorname{listof}\left(M_{1}, x\right) \cdot \text { listof }\left(M_{2}, x\right)
\end{gathered}
$$

Note that in this translation, "P" stands for a (supposed to be unique) string identifier attached to the process $P$. This string informs weaver and advice that they must send a message on the label resume ${ }_{M},{ }$ " to proceed.

\subsection{The weaver}

We define, for each possible pattern $M$ present in a class defined in $C T$, a weaver $W_{M}$ dedicated to $M$. Technically, $M$ is considered up-to consistent renaming of its free variables, that is there is only one weaver for each equivalent class of patterns defined in $C T$. To know which advice can be deployed, the weaver maintains the list of all pieces of advice $A d$ (first argument of adviceList) that have a pointcut that intercepts the rule $M$. The weaver also stores aspect defining advice of $A d$ by the list $A$ (second argument of
adviceList) and the associated pointcut list Pc (third argument of adviceList). Note that pointcuts and advice can not be passed directly as arguments of messages but we take this liberty for clarity (this approximation can be removed by an encoding).

When an aspect sends a message add_advice $(a, p c, a d)$ to register a new advice, the weaver updates advice List accordingly. When the message weave $(x, p, f, H, \vec{v})$ is captured, the weaver tests the validity of the list pointcuts $P c$ of advice described by the list $A d$ by executing

$$
\text { let }(B=\operatorname{test}(P c, f, H)) \text {. }
$$

We do not detail here the test function test as it basically performs the boolean test described in each $P c_{i}^{o p t}$ based on the flow information $f$ and the host information $H$. Note that an other possibility would have been to include the test in each aspect, but then it raises synchronization issue that would have make the translation much harder.

If no advice can be deployed (is_false $(B)$ is true) then the weaver executes the original process by sending the message resume $_{M, p}(f, \vec{v})$ to the object $x$. This corresponds to Rule RED/NO ASP. Otherwise, the weaver asynchronously deploys any applicable advice $A d_{i}$ (that is advice for which $b_{i}$ is true) by sending the message $a_{i}$.deploy $\left.A d_{i}(x, p, f, \vec{v})\right)$ to the associated aspect $a_{i}$. This corresponds to Rule RED/AsP.

The central weaver $W$ is just the parallel composition of all local weavers

$$
W=\bigotimes_{M \in C T} W_{M}
$$

for every pattern $M$ appearing in the class table $C T$.

\subsection{The translation of aspects}




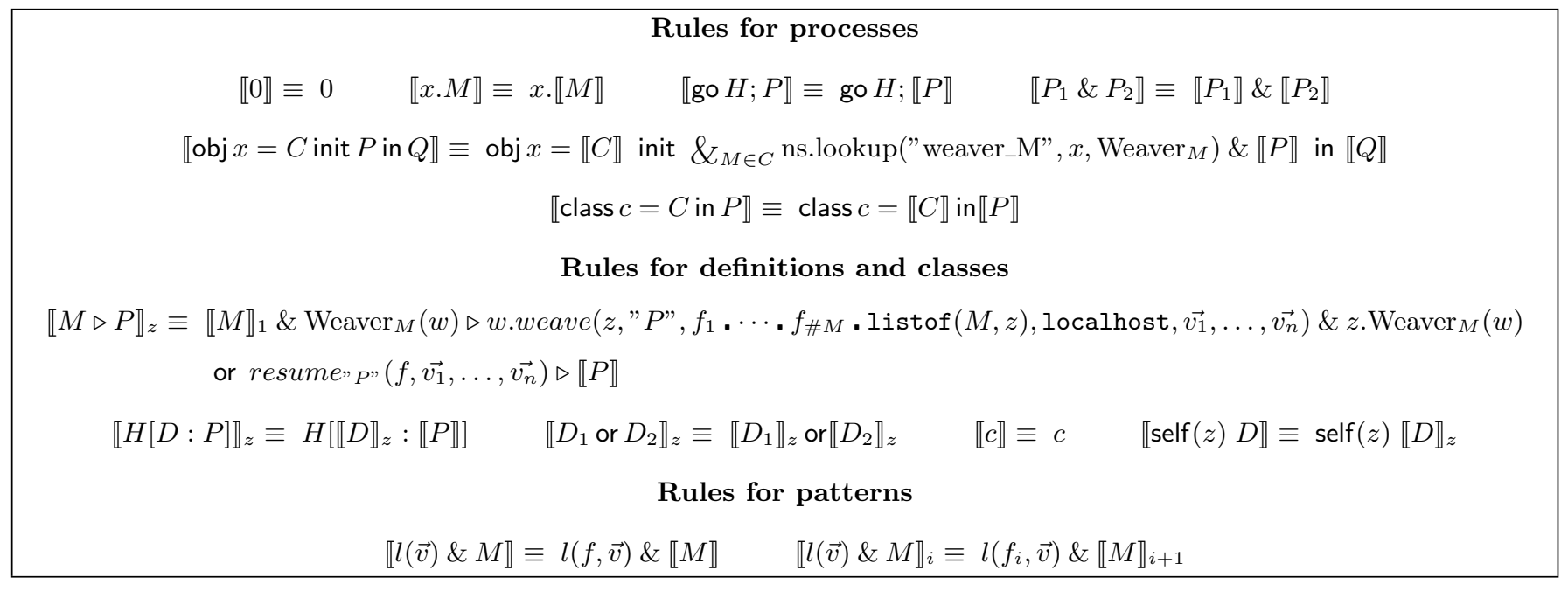

Figure 8: Translation of processes

\begin{tabular}{|c|c|c|}
\hline obj & $W_{M}=$ & $\begin{array}{rr}\text { add_advice }(a d, a, p c) \& \text { adviceList }(A d, A, P c) \triangleright \text { adviceList }(a d: A d, a: A, p c: P c) & \text { (* store new advice } *) \\
\text { weave }(x, p, f, H, \vec{v}) \& \text { adviceList }(A d, A, P c) \triangleright & \text { (* receive join point } *) \\
\text { let }(B=\text { test }(P c, f, H)) \text { in adviceList }(A d, A, P c) \& & \text { (* test applicability of aspects } *) \\
\text { if is_false }(B) \text { then x.resume } M, p(f, \vec{v}) & (* \text { if no aspect, return to join point } *) \\
\text { else }\left(\&_{b_{i} \in B} \text { if } b_{i} \text { then } a_{i} \cdot \operatorname{deploy} A d_{i}(x, p, f, \vec{v})\right) & \left(* \text { for all pieces of advice if } P c_{i}, \text { deploy } A d_{i} *\right) \\
\text { ns.register }\left(W_{M}, " \text { weaver_M") \& adviceList }([],[],[])\right. & (* \text { register weaver_M and initializes lists } *)\end{array}$ \\
\hline
\end{tabular}

Figure 9: Definition of the weaver for pattern $M$

In our translation, an aspect is seen as a classical object that receives messages from the weaver to execute particular methods that represent advice bodies. This is close to the CaesarJ point of view that aspects are just objects that happen to have some pointcuts as attributes [2]. A call to proceed is translated into a message resume $_{M, "} P^{\prime}(f, \vec{v})$ that is sent to the object whose pattern $M$ has been intercepted. More precisely, given an aspect

$$
\text { aspect } A=\operatorname{self}(z) D \text { init } P \text { intercept }\left(P c_{i}\left\{A d_{i}\right\}\right)_{i \in I},
$$

the translation produces the object

$$
\begin{aligned}
\text { obj } a= & \operatorname{self}(z) \llbracket D \rrbracket \\
& \text { or deployAd }(x, p, f, \vec{v}) \triangleright \llbracket A d_{i}\{z / a\} \rrbracket M_{i} \\
& \text { or } \ldots \\
& \text { init } \llbracket P \rrbracket \& A d d(A d, a, P c)
\end{aligned}
$$

where the translation of processes is extending to proceed by

$$
\llbracket \operatorname{proceed}(\vec{v}) \rrbracket_{M} \equiv \text { x.resume } \text { res }, p(f, \vec{v})
$$

The definitions $D$ of the object part of $a$ are extended with reaction rules that deploy an advice $A d_{i}$ when the weaver sends the message $\operatorname{deploy} A d_{i}(x, p, f, \vec{v})$. The initialization sends asynchronously every advice appearing in the definition of the aspect $a$ to its associated weaver by using the dedicated process $A d d(A d, a, P c)$. We do not detail this process here has it is just a matter of bureaucracy using add_advice.

\subsection{Bisimilarity of abstract and concrete defi- nitions}

The main interest of translating the aspect join calculus into the core join calculus is that it provides a direct implementation of the weaving algorithm that can be proved to be correct. As usual in concurrent programming languages, the correctness of the algorithm is given by a proof of bisimilarity. Namely, we prove that the original configuration with aspects is bisimilar to the translated configuration that has no aspect. The idea of bisimilarity is to express that, at any stage of reduction, both configuration can perform the same actions in the future. More formally, in our setting, a simulation $\mathcal{R}$ is a relation between configurations such that when $\mathcal{C}_{0} \mathcal{R} \mathcal{C}_{1}$ and $\mathcal{C}_{0}$ reduces in one step to $\mathcal{C}_{0}^{\prime}$, there exists $\mathcal{C}_{1}^{\prime}$ such that $\mathcal{C}_{0}^{\prime} \mathcal{R} \mathcal{C}_{1}^{\prime}$ and $\mathcal{C}_{1}$ reduces (in 0, 1 or more steps) to $\mathcal{C}_{1}^{\prime}$. We illustrate this with the following diagram

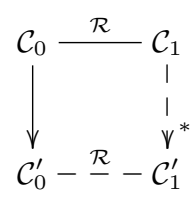

A bisimulation is a simulation whose inverse is also a simulation.

To relate a configuration $\mathcal{C}_{0}$ with its translation $\llbracket \mathcal{C}_{0} \rrbracket$, we need to tackle three difficulties:

1. During the evolution of $\llbracket \mathcal{C}_{0} \rrbracket$, auxiliary messages that have no correspondents in $\mathcal{C}$ are sent for communication between processes, weaver and aspects.

2. In the execution of $\mathcal{C}_{0}$, proceed is substituted by the process $P$ to be executed, whereas in $\llbracket \mathcal{C}_{0} \rrbracket, P$ is exe- 
cuted through a communication with the object where the reaction has been intercepted.

3. Initial communications with the name server in $\llbracket \mathcal{C}_{0} \rrbracket$ disappear during the reduction.

To see the auxiliary communication as part of a reduction rule of the aspect join calculus, we define a notion of standard form for the translated configurations. Let

$$
\mathbb{T}=\left\{\mathcal{C} \mid \exists \mathcal{C}_{0}, \llbracket \mathcal{C}_{0} \rrbracket \longrightarrow{ }^{*} \mathcal{C}\right\}
$$

be the set of configurations that comes from a translated configuration. We construct a rewriting system $\longrightarrow \mathbb{T}$ for $\mathbb{T}$, based on the reduction rule of the join calculus. Namely, we take Rule RED restricted to the case were the pattern contains either of the dedicated labels: weave, resume, $\operatorname{deploy} A d$, reply $M$ or add_advice. In $\mathbb{T}$, those labels only interact one-by-one with constant labels (a constant label is a label that appears identically on the left and right hand side of every reaction rule) such as Weaver or aspL(). So the order in which reaction rules are selected has no influence on the synchronized pattern, that is the rewriting system $\longrightarrow_{\mathbb{T}}$ is confluent. Furthermore, it is not difficult to check that this rewriting system is also terminating. Therefore, it makes sense to talk about the normal form of $\mathcal{C} \in \mathbb{T}$, noted $\widetilde{\mathcal{C}}$.

We note $\mathcal{C} \stackrel{\text { proc }}{\sim} \mathcal{C}^{\prime}$ when $\mathcal{C}^{\prime}$ is equal to $\mathcal{C}$ where every message resume $_{M, "} P,(f, \vec{v})$ is substituted by the process $P(\vec{v})$.

Given a configuration $\mathcal{C}_{0}$, we note $\llbracket \mathcal{C}_{0} \rrbracket_{\text {init }}$ the translated configuration where every initial communication with the name server has been performed. That is, every message of the form ns.lookup(" $a ", x, l)$ and ns.register $(a$, ,a") have been consumed.

ThEOREM 3. The relation $\mathcal{R}=\left\{\left(\mathcal{C}_{0}, \mathcal{C}_{1}\right) \mid \widetilde{\mathcal{C}_{1}} \stackrel{\text { proc }}{\sim} \llbracket \mathcal{C}_{0} \rrbracket_{\text {init }}\right\}$ is a bisimulation. In particular, any typed configuration is bisimilar to its translation.

Proof. The fact that $\mathcal{R}$ is a simulation just says that the communication between aspects, processes and the weaver simulates the abstract semantics of aspects. More precisely, we show that for any reduction $\mathcal{C}_{0} \longrightarrow \mathcal{C}_{0}^{\prime}$ using Rule RED/AsP, $\mathrm{RED} / \mathrm{NO} \mathrm{ASP}$ or ADV, one can find a corresponding reduction chains from $\llbracket \mathcal{C}_{0} \rrbracket_{\text {init }}$ to $\llbracket \mathcal{C}_{0}^{\prime} \rrbracket_{\text {init }}$

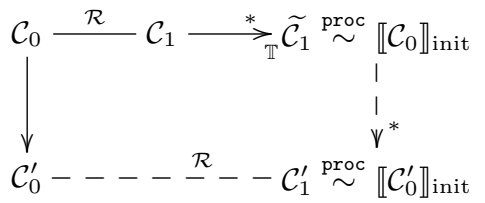

Consider the case of Rule RED/AsP (the others are easier):

$$
x . l(\vec{v}) \quad \longrightarrow \quad A d_{1}[P / \text { proceed }] \& \cdots \& A d_{n}[P / \text { proceed }]
$$

This rule is simulated by the chain (we omit argument on the right for saving place)

$$
\begin{array}{rll}
x . l(f, \vec{v}) & \longrightarrow & \text { w.weave } \\
& \longrightarrow & a_{1} \cdot \text { deploy } A_{1} \& \cdots \& a_{n} . \operatorname{deploy} A d_{n} \\
& \longrightarrow & \llbracket A d_{1}\{z / a\} \rrbracket_{A} \& \cdots \& \llbracket A d_{n}\{z / a\} \rrbracket_{A}
\end{array}
$$

leading the normal form $\mathcal{C}_{1}^{\prime} \stackrel{\text { proc }}{\sim} \llbracket \mathcal{C}_{0}^{\prime} \rrbracket_{\text {init }}$.
The converse direction is more interesting as it says that any reduction in the translated configuration can be seen as a the activation of advice (Rule ADV) or as a step in the simulated reduction of a Rule RED/ASP or RED/NO ASP of the original configuration. More precisely, we have to show that any reduction $\mathcal{C}_{1} \longrightarrow \mathcal{C}_{1}^{\prime}$ can be seen as a reduction between their normal forms. This expressed by the following diagram:

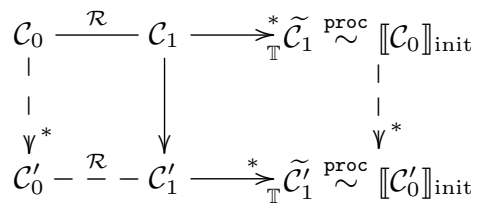

If the reduction is $\mathcal{C}_{1} \longrightarrow \mathbb{T} \mathcal{C}_{1}^{\prime}$, then $\widetilde{\mathcal{C}_{1}}=\widetilde{\mathcal{C}_{1}^{\prime}}$ and $\mathcal{C}_{0}=\mathcal{C}_{0}^{\prime}$. If it introduces a message resume ${ }_{M}, " P^{\prime \prime}(f, \vec{v})$, then $\widetilde{\mathcal{C}_{1}^{\prime}} \stackrel{\text { proc }}{\sim} \widetilde{\mathcal{C}_{1}} \stackrel{\text { proc }}{\sim}$ $\llbracket \mathcal{C}_{0} \rrbracket_{\text {init. }}$. If it introduces a message add_advice $(a d, a, p c)$, then $\mathcal{C}_{0} \stackrel{A d v}{\longrightarrow} \mathcal{C}_{0}^{\prime}$ and $\widetilde{\mathcal{C}_{1}^{\prime}} \stackrel{\text { proc }}{\sim} \llbracket \mathcal{C}_{0}^{\prime} \rrbracket_{\text {init }}$. Otherwise, the reduction consumes a pattern $x . M_{\sigma}$ and produces a message of the form

$$
\text { w.weave }(z, " P ", f, \text { localhost }, \vec{v}) \text {. }
$$

Then, if some aspects can be deployed, $\mathcal{C}_{0}^{\prime}$ is obtained by applying Rule RED/AsP to $x \cdot M \triangleright P \Vdash^{\varphi} x \cdot M_{\sigma}$, and if no aspect can be deployed, $\mathcal{C}_{0}^{\prime}$ is obtained by applying Rule RED/No AsP to $x . M \triangleright P \Vdash^{\varphi} x . M_{\sigma}$. The fact that the diagram above commutes is a direct consequence of the confluence of $\longrightarrow \mathbb{T}$ and its non-interference with other reductions of the system.

We conclude the proof of the theorem by noting that $\llbracket \mathcal{C}_{0} \rrbracket_{\text {init }}$ is a normal form for $\longrightarrow \mathbb{T}$, so that $\mathcal{C}_{0} \mathcal{R} \llbracket \mathcal{C}_{0} \rrbracket_{\text {init }}$.

The crux of the proof lies in the confluence of $\longrightarrow \mathbb{T}$ which means that once the message weave $(x, p, f, H, \vec{v})$ is send to the weaver, the translation introduces no further choice in the configuration. That is, every possible choice in $\llbracket \mathcal{C} \rrbracket$ corresponds directly to the choice of a reduction rule in $\mathcal{C}$.

Note that the bisimulation we have defined is not barbedpreserving nor context-closed. This is not surprising as a context would be able to distinguish between the original and translated configuration by using the flow information. But we are interested in equivalent behaviour of two closed configurations, not of two terms that can appear in any context, so a simple bisimulation is sufficient in our case.

\section{CONNECTIONS TO EXISTING DISTRIBUTED AOP SYSTEMS}

Local weavers. In our implementation of the weaving algorithm, we have made the choice of a central weaver as in DJCutter [12]. Even if this was enough for the correction of the algorithm, it might be inefficient from a practical point of view as every machine would have to connect to the same server, this resulting in a network congestion. A more realistic algorithm would be to attach each local weaver and its corresponding aspects to the location where the intercepted method will be executed. This implementation would be closer to the decentralized architecture of AWED [11] and ReflexD [17]. 
Migration of aspects and advice. In the aspect join calculus, one can attach an aspect to an object. It suffices to host the aspect at a sub-location of the object, and thus the aspect will migrate with its object. The property that an aspect is attached to an object has been discussed in [16].

Note that it also possible to define more general migration of aspects and advice. For example, one can define an aspect with an advice that migrates at each deployment to an host that possesses the resource of interest.

Grouping host. In AWED and ReflexD, there is a notion of group of hosts that can be dynamically managed by adding and removing host. A group of hosts can then be used to define pointcuts. This mechanism can be translated in the aspect join calculus by a creation of a location for each group of hosts and a migration of the hosts to that location. Adding or removing an host will also be performed by migration. Then, a pointcut can be defined on the location of the group. Nevertheless, this point of view is less general than the mechanism used in AWED and ReflexD as it presents the drawback to force a tree-like structure for the configuration of hosts.

Synchronous aspects in sequence. Our deployment of aspects is, as the join calculus, eminently asynchronous. Nevertheless, we can encode sequential execution by adding a channel a.proceedAd and a definition

$$
\operatorname{proceed} \operatorname{Ad}(\vec{v}) \triangleright \operatorname{proceed}(\vec{v})
$$

for every advice $A d$ of an aspect $a$. A normal call to proceed in $A d$ is then replaced by a call to $a$.proceedAd. We will use those new labels to trigger the execution of aspects. Suppose that two pieces of advice $P c_{1}\left\{A d_{1}\right\}$ and $P c_{2}\left\{A d_{2}\right\}$ interrupt the same method c.M. Then, in traditional synchronous setting, the user has to define the order of execution between both aspects, let say $P c_{1}\left\{A d_{1}\right\}$ before $P c_{2}\left\{A d_{2}\right\}$. In the aspect join calculus, we will define two pieces of advice for translating $P c_{2}\left\{A d_{2}\right\}$ : one that triggers c. $M$ with the optional condition that $P c_{2}^{\text {opt }} \wedge \neg P c_{1}^{\text {opt }}$ is satisfied, and one that triggers the call to proceed of $P C_{1}\left\{A d_{1}\right\}$ with the optional argument that $P c_{2}^{o p t}$ is satisfied.

$$
P c_{2}\left\{A d_{2}\right\} \Rightarrow\left\{\begin{array}{l}
\operatorname{rule}(c \cdot M(\vec{v})) \wedge P c_{2}^{o p t} \wedge \neg P c_{1}^{o p t}\left\{A d_{2}\right\} \\
\operatorname{rule}\left(a_{1} \cdot \operatorname{proceed} \operatorname{Ad}(\vec{v})\right) \wedge P c_{2}^{o p t}\left\{A d_{2}\right\}
\end{array}\right.
$$

Note that the coding of sequential aspects induces an exponential increase in the number of pieces of advice.

After and Before advice. In an asynchronous setting, After and Before advice à la Aspect $J$ do not really make sense. Nevertheless, in an synchronous setting where every method has an entry point and a return value, those two kind of advice can be easily encoded. Before is encoded by an advice that intercepts the call of a method $m$ and where exactly one proceed is performed at the end of the advice body

$$
\text { before }(c . m)\{P\} \equiv \text { intercept } \operatorname{rule}(c . m(\vec{v}))\{P ; \operatorname{proceed}(\vec{v})\}
$$

In the same way, After is encoded by an advice that intercepts the return label of the method and where exactly one proceed is performed at the beginning of the advice body.
Call and execute pointcuts. We can not interpret the difference between call and execute pointcuts in our setting. This is due to the absence of inheritance in our model. But as we have already said, inheritance can be added smoothly following the work of $[6]$.

Distributed control flow. In the objective join calculus, there is no notion of method body and return value. The flow of execution just indicates that a method has been called, but says nothing about termination. Then, the pointcut flow $(l)$ just says that $l$ has been called at least once during the reduction, an information that appears quickly to be useless. Nevertheless, in a synchronous setting, we can extract the traditional notion of control flow from this "flat" notion of flow already present in the calculus. Indeed, when every method has a call and return discipline, we can parse the flow of execution and detect the called methods that have not returned yet. More precisely, with the continuation passing encoding, a call $x . m(k, \vec{v})$ to method $x . m$ has not returned when the message $k$.return $(\vec{w})$ does not appear after in the flow. This enables to define the well-known AspectJ pointcut designator Cflow. In the same way, we can construct a within pointcut designator.

Changing the route of messages. A common use of aspects in a distributed setting is the re-routing of messages. For example, one would like to intercept and re-route every message sent to a machine that has crashed. This interception of message is not directly possible in the aspect join calculus. This is because routing of messages remains implicit and does not constitute an observable event of the language. However, we can recover routing information after the following encoding. Any emission of the message $y . l(\vec{v})$ by an object $x$ is replaced by the emission of the message $x$.send_l $l(y, \vec{v})$ and the reaction rule

$$
\operatorname{send} \_l(y, \vec{v}) \triangleright y \cdot l(\vec{v}) .
$$

Then, an aspect can prevent routing of the message $y . l$ to the host were $y$ is situated by intercepting the message $x . s e n d \_l(y, \vec{v})$.

\section{CONCLUSIONS}

This paper provides a formal theory of distributed aspects. Based on the distributed and objective join calculus, our calculus is presented with a (chemical) operational semantics and a type system. This type system guarantees safety properties such as the absence of mismatch in the number or type of arguments when an aspect returns to the original program using the keyword proceed, or the absence of host duplication in the network.

We have also defined a translation of the aspect join calculus into the core join calculus and shown the correctness of the translation with a proof of bisimilarity. In this way, we provide a well-defined version of a weaving algorithm that constitutes the main step towards an implementation of the aspect join calculus directly in JoCaml.

This paper has also shown that the main features of previous distributed AOP systems can be modeled by the few relatively simple constructs available in the aspect join calculus. Those key features are: remote pointcuts, distributed advice, migration of aspects, asynchronous and synchronous aspects, re-routing of messages and distributed control flow. 


\section{ACKNOWLEDGMENTS}

The author wants to thank Rémi Douence, Hervé Graal, Jacques Noyé and Mario Südholt for valuable discussions and comments.

\section{REFERENCES}

[1] A. Ahern and N. Yoshida. Formalising Java RMI with explicit code mobility. Theoret. Comput. Sci., 389(3):341-410, 2007.

[2] I. Aracic, V. Gasiunas, M. Mezini, and K. Ostermann. An Overview of CaesarJ. Transactions on AOSD I, 3880:135-173, 2006.

[3] B. De Fraine, M. Südholt, and V. Jonckers. StrongAspectJ: flexible and safe pointcut/advice bindings. In 7th conference on AOSD, pages 60-71, 2008.

[4] C. Fournet and G. Gonthier. The reflexive CHAM and the join-calculus. In 23th symposium on POPL, pages 372-385, 1996.

[5] C. Fournet and G. Gonthier. The join calculus: a language for distributed mobile programming. Lecture Notes in Comput. Sci., 2395:268-332, 2002.

[6] C. Fournet, C. Laneve, L. Maranget, and D. Remy. Inheritance in the join calculus. J. Logic Alg. Programming, 57(1):23-70, 2003.

[7] A. Igarashi, B. Pierce, and P. Wadler. Featherweight Java: A minimal core calculus for Java and GJ. Transactions on PLS, 23(3):396-450, 2001.

[8] R. Jagadeesan, A. Jeffrey, and J. Riely. A calculus of untyped aspect-oriented programs. In Proceedings of ECOOP, pages 54-73. Springer-Verlag, 2003.

[9] A. Jeffrey. A distributed object calculus. In Proceedings of the 25th workshop on foundations of object-oriented languages, 2000.

[10] G. Kiczales, E. Hilsdale, J. Hugunin, M. Kersten, J. Palm, and W. G. Griswold. An Overview of AspectJ. In Proceedings of the 15th European Conference on Object-Oriented Programming, pages 327-353, 2001.

[11] L. Navarro, M. Südholt, W. Vanderperren, B. De Fraine, and D. Suvee. Explicitly distributed AOP using AWED. In 5th conference on AOSD, pages 51-62, 2006.

[12] M. Nishizawa, S. Chiba, and M. Tatsubori. Remote pointcut: a language construct for distributed AOP. In 3rd conference on AOSD, pages 7-15, 2004.

[13] R. Pawlak, L. Seinturier, L. Duchien, G. Florin, F. Legond-Aubry, and L. Martelli. JAC: an aspect-based distributed dynamic framework. Software: Practice and Experience, 34(12), 2004.

[14] J. Riely and M. Hennessy. A typed language for distributed mobile processes. In 25th symposium on POPL, pages 378-390, 1998.

[15] A. Schmitt. Safe Dynamic Binding in the Join Calculus. In Proc. IFIP TCS, pages 563-575, 2001.

[16] É. Tanter, J. Fabry, R. Douence, J. Noyé, and M. Südholt. Expressive scoping of distributed aspects. In 8th conference on AOSD, pages 27-38, 2009.

[17] E. Tanter and R. Toledo. A versatile kernel for distributed AOP. Lecture Notes in Comput. Sci., 4025:316-331, 2006.
[18] D. Walker, S. Zdancewic, and J. Ligatti. A theory of aspects. In 8th international conference on ICFP, volume 38, pages 127-139, 2003. 